\title{
Changes in Cerebral Glucose Metabolism in Patients with Parkinson Disease with Dementia After Cholinesterase Inhibitor Therapy
}

\author{
Phil Hyu Lee*1, Seok Woo Yong*2, and Young-Sil $\mathrm{An}^{3}$ \\ ${ }^{I}$ Department of Neurology, Yonsei University College of Medicine, Seoul, South Korea; ${ }^{2}$ Department of Neurology, Ajou University \\ School of Medicine, Suwon, South Korea; and ${ }^{3}$ Department of Nuclear Medicine, Kyung Hee University School of Medicine, Seoul, \\ South Korea
}

We investigated changes in cerebral glucose metabolism after cholinesterase inhibitor (ChEl) therapy in patients with Parkinson disease dementia (PDD) to determine whether cognitive improvements would be reflected in changes of cerebral metabolic patterns, thus offering insight into the neural substrate of cognitive dysfunction in patients with PDD. Methods: We performed a serial PET study before (baseline) and after ChEl therapy on 10 patients with PDD, using statistical parametric mapping. Additionally, covariance analysis was performed to extract regions in which increased change in regional cerebral metabolism correlated significantly with increased Mini-Mental State Examination scores. Results: The statistical parametric mapping analysis indicated that significantly increased cerebral metabolism after $\mathrm{ChEl}$ therapy, compared with at baseline, was most evident in the left angular gyrus extending to the supramarginal area and left superior and middle frontal gyri. Additionally, cerebral metabolism was significantly increased in the right superior frontal and left middle orbitofrontal gyri. In contrast, the right fusiform gyrus showed significantly decreased metabolism after ChEl, compared with at baseline. In the correlation analysis, improvements in Mini-Mental State Examination scores after ChEl treatment were significantly associated with increased cerebral metabolism in the left supramarginal, orbitofrontal, and cingulate areas. Conclusion: Our data suggest that prefrontal and parietal association areas may be relevant structures for the pharmacologic response to ChEl in patients with PDD.

Key Words: neurology; PET; cerebral metabolism; Parkinson disease dementia; cholinesterase inhibitor

J Nucl Med 2008; 49:2006-2011

DOI: 10.2967/jnumed.108.054668

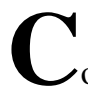

ognitive dysfunction occurs in $25 \%-30 \%$ of patients with Parkinson disease (PD); this prevalence is 6 times greater than that among the population in general (1-3). The

\footnotetext{
Received May 25, 2008; revision accepted Sep. 2, 2008.

For correspondence or reprints contact: Phil Hyu Lee, Department of Neurology, Yonsei University College of Medicine, 134 Shinchon-dong, Seodaemun-gu, Seoul 120-752, South Korea.

E-mail: phisland@chol.com

${ }^{*}$ Contributed equally to this work.

COPYRIGHT $\odot 2008$ by the Society of Nuclear Medicine, Inc.
}

key feature of dementia in PD is executive dysfunction; thus, patients with PD dementia (PDD) have difficulty in tasks that require generation of mental sets, planning, and cognitive sequencing. Additionally, patients with PDD often exhibited worse performance in visuospatial tasks and memory impairment in free recalls, which improved significantly with external cues, suggesting that these patients still had the ability to store new information $(3,4)$.

Although the exact pathophysiologic substrate in PDD is still controversial, progressive involvement of subcortical and cortical structures, by Lewy-type pathology or Alzheimer disease-like histologic changes, has been suggested $(5,6)$. Regarding neurochemical substrates in PDD, recent pathologic and functional studies have suggested that cholinergic deficits were significantly correlated with cognitive dysfunction in patients with PD (7-9). Various kinds of cholinesterase inhibitors (ChEIs), including rivastigmine (10), donepezil (11), and galantamine (12), have proven to be effective in improving cognitive function in patients with PDD, supporting this theory.

Only 1 study, which used SPECT, reported that ChEIs significantly increased cerebral perfusion in frontal and cingulate areas (13). However, images obtained with PET have much greater spatial resolution and attenuation correction than those obtained with SPECT. A study comparing PET and SPECT images in patients with dementia with Lewy bodies (DLB) showed the superiority of PET in demonstrating regional effects in cerebral metabolism (14). In the current study, we performed a serial PET study analysis before and after ChEI therapy in patients with PDD. We used statistical parametric mapping (SPM) to evaluate whether cognitive improvements were reflected in cerebral metabolism after ChEI therapy, thus offering insight into the neural substrate of cognitive dysfunction in patients with PDD.

\section{MATERIALS AND METHODS}

\section{Subjects}

Participants were recruited consecutively from a university hospital outpatient clinic. We prospectively enrolled 12 patients with a clinical diagnosis of PDD. PD was determined according to 
the clinical diagnostic criteria of the UK Parkinson's Disease Society Brain Bank (15). PDD was diagnosed when the patient had dementia due to PD, according to the DSM-IV criteria (16). Patients who developed dementia more than $1 \mathrm{y}$ after the onset of parkinsonian motor symptoms, according to the 1-y rule between the onset of dementia and parkinsonism (17), were included in the study. The severity of dementia was assessed using the MiniMental State Examination (MMSE). Exclusion criteria included evidence of focal brain lesions by MRI, the presence of other neurodegenerative diseases that may account for the dementia, and prior use of a ChEI or anticholinergic drug. Possible medical comorbidities were also excluded by laboratory tests, including a thyroid function test, vitamin B12 and folic acid level assessment, and a venereal disease research laboratory test.

All patients with PDD underwent a cardiac ${ }^{123}$ I-metaiodobenzylguanidine ( ${ }^{123} \mathrm{I}-\mathrm{MIBG}$ ) scan to possibly exclude other conditions of parkinsonism. Patients were treated with donepezil, starting at $5 \mathrm{mg}$ daily for $4 \mathrm{wk}$, which was increased to $10 \mathrm{mg}$ daily. Of 12 patients initially diagnosed with PDD, 2 were excluded in this analysis; one could not tolerate donepezil; and was treated with galantamine, and the other had the delayed heart-to-mediastinum ratio that did not lie more than 2 SDs below the normal mean of our hospital (18). The mean delayed cardiac ${ }^{123}$ I-MIBG uptake in patients with PDD included in this study was 1.23. All subjects gave informed consent to the study, and the trial was approved by the Institutional Review Board for Human Investigation of Ajou University Hospital, Suwon, South Korea.

\section{${ }^{18}$ F-FDG PET Data Acquisition}

In all subjects, the first PET scan was performed before ChEI therapy; the second PET scan was performed when patients had been treated with ChEI therapy for longer than $3 \mathrm{mo}$. PET/CT data were acquired on a Discovery ST scanner (GE Healthcare). Dopaminergic medications were discontinued for more than $12 \mathrm{~h}$ before the PET scan. After fasting for at least $4 \mathrm{~h}$, patients received 300 $\mathrm{MBq}$ of ${ }^{18} \mathrm{~F}$-FDG intravenously. We checked serum glucose levels in all subjects before the ${ }^{18} \mathrm{~F}-\mathrm{FDG}$ injection; subjects whose glucose level exceeded $150 \mathrm{mg} / \mathrm{dL}$ were excluded. All subjects were instructed to rest comfortably for $30 \mathrm{~min}$ with their eyes closed and ears unplugged, and then image acquisition was started. To reduce head movement during scanning, patients were positioned and maintained using an individually molded head holder. Patients first underwent a CT scan (tube rotation time, $1 \mathrm{~s} /$ revolution; $120 \mathrm{kV}$;
$70 \mathrm{~mA} ; 5.0 \mathrm{~mm} /$ rotation; acquisition time, $11.8 \mathrm{~s}$; scan length, $150.42 \mathrm{~mm}$ ). Subsequently, emission PET data (1 frame, $8 \mathrm{~min} /$ frame) were acquired in 3-dimensional mode. PET images were reconstructed by iterative reconstruction (ordered-subsets expectation maximization, with 1 iteration and 32 subsets), using the CT images for attenuation correction. The randoms correction by singles and model-based scatter correction was also applied.

\section{Data Analysis}

${ }^{18}$ F-FDG PET images were spatially normalized to a standard template provided by SPM2 (Institute of Neurology, University of London) in MATLAB (version 7.1; Mathworks Inc.). A local optimization of the 12 parameters of an affine transformation was applied to the spatial normalization. The images were then smoothed with a gaussian kernel (full width at half maximum, $16 \mathrm{~mm}$ ) to minimize noise and improve between-subject spatial alignment. To identify brain regions in which metabolic activity had changed after ChEI therapy, baseline and follow-up PET images were compared voxel by voxel (2-tailed paired $t$ test), and an uncorrected $P$ value of less than 0.005 was considered significant. Additionally, covariance analysis was performed to extract regions in which increased changes in regional cerebral metabolism were significantly correlated with the increase in MMSE scores, using a single-subject covariate model. Regions reaching an uncorrected $P$ value of less than 0.05 were considered significant in the covariance analyses. Anatomic labeling of significant voxels was performed using the automated anatomic labeling SPM toolbox (19), which was based on anatomy provided by the Montreal Neurologic Institute.

\section{Statistical Analysis}

A Wilcoxon paired signed rank test was used to compare the changes in MMSE scores after ChEI therapy; $P$ values of less than 0.05 were deemed statistically significant. Statistical analyses were performed using commercially available software (SPSS, version 13.0; SPSS Inc.).

\section{RESULTS}

The characteristics of the patients are summarized in Table 1. Data are mean \pm SD. The mean age of the patients was $74.7 \pm 4.5 \mathrm{y}$. The mean disease durations of PD and PDD were $6.4 \pm 4.2 \mathrm{y}$ (range, $2-14 \mathrm{y}$ ) and $8.4 \pm 6.4 \mathrm{mo}$

TABLE 1

Demographic Characteristics in Patients with PDD

\begin{tabular}{|c|c|c|c|c|c|c|c|}
\hline Patient no. & Sex & Age $(y)$ & $\begin{array}{c}\text { Dopaminergic } \\
\text { drug }(\mathrm{mg})\end{array}$ & $\begin{array}{c}\text { PD } \\
\text { duration (y) }\end{array}$ & $\begin{array}{c}\text { Dementia } \\
\text { duration }(\mathrm{mo})\end{array}$ & $\begin{array}{c}\text { First } \\
\text { MMSE }\end{array}$ & $\begin{array}{l}\text { Second } \\
\text { MMSE }\end{array}$ \\
\hline 1 & $M$ & 81 & L 600/R 6 & 14 & 4 & 15 & 21 \\
\hline 2 & $\mathrm{~F}$ & 78 & L 450 & 5 & 24 & 22 & 19 \\
\hline 3 & $M$ & 75 & L 600 & 3 & 6 & 16 & 18 \\
\hline 4 & $\mathrm{~F}$ & 68 & L 450/E 600 & 3 & 3 & 21 & 29 \\
\hline 5 & $\mathrm{~F}$ & 67 & L 450 & 2 & 5 & 20 & 25 \\
\hline 6 & $\mathrm{~F}$ & 77 & L 600/E 800 & 10 & 3 & 16 & 27 \\
\hline 7 & M & 78 & L 600/E 800 & 3 & 6 & 19 & 23 \\
\hline 8 & $\mathrm{~F}$ & 77 & L 450 & 10 & 9 & 17 & 22 \\
\hline 9 & $M$ & 73 & L 450/E 600 & 4 & 12 & 23 & 25 \\
\hline 10 & $M$ & 73 & L 700/E 800 & 10 & 12 & 22 & 30 \\
\hline
\end{tabular}

$\mathrm{L}=$ levodopa; $\mathrm{R}$ = ropinirole; $\mathrm{E}=$ entacapone. 
(range, 3-24 mo), respectively. The mean stage of Hoehn and Yahr was $3.1 \pm 0.4$. The mean duration of education was $6.6 \pm 5.3 \mathrm{y}$. Two patients were current smokers. The mean interval between the first and second PET scans was $3.5 \pm 0.3$ mo. The mean MMSE score at baseline was $19.1 \pm$ 2.9 (range, 15-23). After ChEI therapy (mean treatment duration, $3.5 \mathrm{mo}$ ), a significant improvement was observed in MMSE scores $(23.9 \pm 4.0$; mean change, 4.8 points; $P=$ 0.012 ). In analyzing the change of subitems of MMSE before and after ChEI therapy, serial sevens and delayed recall subitems were significantly improved after ChEI treatment (each mean change of score, 1.0 and 1.4, respectively; each $P=0.04$ and 0.01 , respectively). The registration subitem was marginally improved (mean change of score, $0.5 ; P=0.09$ ). However, no significant improvement in other subitems of time orientation ( 0.7 points), place orientation ( 0.4 points), language ( 0.8 points), and pentagon copy ( 0 point) was demonstrated.

The SPM analysis showed that significantly increased cerebral metabolism after ChEI therapy, compared with at baseline, was most evident in the left angular gyrus extending to the supramarginal gyrus and superior and middle frontal gyri $\left(P_{\text {uncorrected }}<0.001\right)$. Additionally, cerebral metabolism was significantly increased in the right superior frontal and left middle orbitofrontal gyri $\left(P_{\text {uncorrected }}<0.005\right.$; Fig. 1; Table 2). In contrast, the right fusiform gyrus showed significantly decreased cerebral metabolism after ChEI therapy, compared with baseline $\left(P_{\text {uncorrected }}<0.001\right.$; Fig. 2; Table $2)$. In the correlation analysis between changes in cerebral metabolism and MMSE scores, improved MMSE scores after ChEI treatment were significantly associated with increased cerebral metabolism in the left supramarginal, left orbitofrontal, and left cingulate cortices $\left(P_{\text {uncorrected }}<0.05\right.$, Fig. 3; Table 3).

\section{DISCUSSION}

Previous postmortem and in vivo studies demonstrated that cholinergic activity in patients with PDD was profoundly lowered in the cerebral cortex, showing more severe cholinergic dysfunction in patients with PDD than in patients with Alzheimer disease (AD) $(8,20,21)$. With

FIGURE 1. Statistical parametric maps showing spatial distribution of significant metabolic increase after ChEl therapy in patients with PDD. patients with $\mathrm{AD}$, there were reports about the effects of both short-term and long-term treatment of ChEI on the change of cerebral metabolism (22-24); however, no study has reported on changes in cerebral metabolic patterns after ChEI use in patients with PDD. In this study, we evaluated the cerebral metabolic pattern before and after ChEI treatment and correlated cerebral metabolism with cognitive improvement using SPM analysis. We demonstrated that cognitive function as measured by MMSE scores significantly improved after the introduction of ChEIs, compared with at baseline, and cerebral metabolism increased significantly in the prefrontal and left parietal association areas. In a correlation analysis involving the change in cerebral metabolism and MMSE scores, cognitive improvement after ChEI therapy was significantly associated with increased cerebral metabolism in the left supramarginal, orbitofrontal, and cingulate cortices.

In our study, the cortical area showing increased metabolism after ChEI treatment was consistent with regions of decreased cholinergic activity in patients with PDD, compared with those with PD. Hilker et al. (25), in an in vivo study using $N-{ }^{11} \mathrm{C}$-methyl-4-piperidyl, an acetylcholine analog, reported that regional acetylcholine esterase activity significantly decreased in the parietal and limbic areas in patients with PDD, compared with those with PD without dementia. Furthermore, the increased metabolic area after ChEI treatment is consistent with the cortical area showing decreased perfusion or metabolism in PDD. Firbank et al. (26) demonstrated that patients with PDD showed more perfusion deficits in the lateral parietal association area than did patients who had PD without dementia. Recently, in a study of metabolic patterns using SPM analysis, we demonstrated that patients with PDD, compared with patients with PD, showed patterns of decreased metabolism in the bilateral inferior and medial frontal lobes and in the right parietal lobe (27). For the correlation of cognitive status with choline acetyltransferase activity and cerebral perfusion in patients with PDD, choline acetyltransferase activity in the prefrontal cortex and cerebral perfusion in the parietal cortex had a significant negative correlation with cognitive impairment (7). Additionally, several studies have demonstrated that ChEI treatment significantly improved attentional, executive, and visuospatial functions $(3,28)$, suggesting a potential role for ChEIs in refining control in frontal and parietal function. Although the MMSE is not sensitive in detecting executive and visuospatial functions, cognitive improvement after ChEI in our study seems to be related to attentionmediated MMSE subitems (serial sevens, delayed recall, and, partially, registration). Our data, showing increased metabolism in the prefrontal and left parietal association areas and a significant correlation between changes in metabolism and cognitive improvement, suggest that these areas may be relevant structures for the pharmacologic response to $\mathrm{ChEI}$ therapy in PDD. Our results relate to findings that the degree of cerebral metabolism was significantly correlated with acetylcholine esterase activity in patients with dementia (29). 
TABLE 2

Brain Area Showing Significant Change in Glucose Metabolism After ChEl Therapy in Patients with PDD

\begin{tabular}{ccccll}
\hline $\begin{array}{c}\text { Glucose } \\
\text { metabolism }\end{array}$ & \multicolumn{3}{c}{ Coordinate } & & \\
\cline { 2 - 4 } Increased & -50 & -54 & 28 & Left & Angular gyrus extending to supramarginal gyrus \\
& -20 & 20 & 44 & Left & Superior and middle frontal gyrus \\
& -26 & 54 & -4 & Left & Middle orbitofrontal gyrus \\
& 18 & 40 & 36 & Right & Superior frontal gyrus \\
Decreased & 32 & -30 & -18 & Right & Fusiform gyrus extending to parahippocampal gyrus \\
\hline
\end{tabular}

The metabolic or perfusion pattern in response to ChEI therapy seems to be different between PDD and DLB. Mori et al. (30) found that increased perfusion after ChEI treatment in patients with DLB was localized in the occipital area, whereas a previous study by Ceravolo et al. (13) and our current data showed that increased perfusion or metabolism in patients with PDD was observed in the frontal or parietal areas. Controversy still exists as to whether PDD and DLB are indeed the same disease entities. The characteristics of cognitive dysfunction and the pattern of metabolic derangement are similar between the 2 conditions, although these patterns tend to be more severe in DLB than in PDD. However, recent functional imaging and neuropathologic data demonstrated that the burden of AD-like pathology and the density of Lewy bodies are more severe in DLB than in PDD $(6,31,32)$, suggesting different pathologic backgrounds for the 2 diseases. Thus, on the basis of our data and those of another study, we speculate that at least the anatomic substrate for the response to ChEIs in patients with PDD may be different from those with DLB, although this difference does not directly indicate a different nature of disease between PDD and DLB.

The increased metabolism in the frontal and parietal regions after ChEI treatment in our study looks similar to the short-term perfusion change pattern seen in response to ChEIs in patients with $\mathrm{AD}$, except for the metabolic or perfusion changes in the temporal area $(33,34)$. Because all patients in this study had a lower cardiac MIBG uptake score

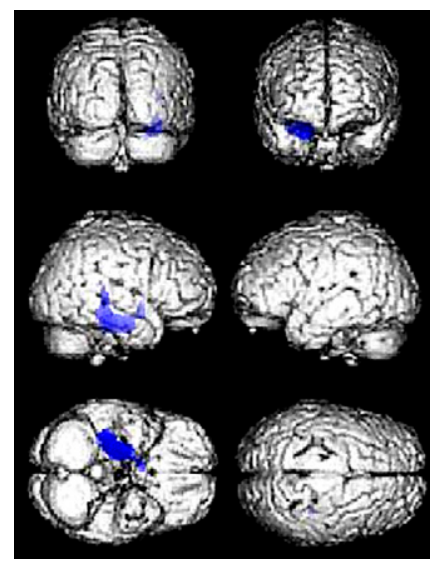

FIGURE 2. Statistical parametric maps showing spatial distribution of significant metabolic decrease after ChEl therapy in patients with PDD. (in the $\mathrm{PD}$ range at our institute), and patients with $\mathrm{AD}$ are known to have a reference range of cardiac MIBG uptake (35), it is less likely that patients with AD would have been included in our study. Nevertheless, without autopsy findings it is impossible to exclude coexisting AD pathology in our patients. In vivo imaging using ${ }^{11} \mathrm{C}$-PIB may be useful in excluding coexisting AD pathology because PDD had a lower burden of amyloid in the cortical area than did AD (32). A possible explanation is that the restorative capacity of ChEI therapy may focus particularly on attention and executive functions by enhancing cholinergic neurotransmission in certain areas of the frontal cortex, regardless of the underlying pathologic process, thus resulting in similar changes in functional images. However, this possibility is difficult to reconcile with perfusion change localized in the occipital area in patients with DLB. In addition to the pathologic status of the nucleus basalis of Meynert, cortical cholinergic activity seems to be influenced by the type of protein aggregates (20). Thus, a further direct comparative study regarding the effect of ChEI therapy on changes in functional imaging between patients with PDD, DLB, and AD would be needed to clarify whether response patterns in functional imaging after ChEI therapy are different with different pathologic status.

Interestingly, a significantly decreased metabolism after ChEI treatment in this study was localized in the fusiform gyrus. It has been suggested that the fusiform gyrus is strongly involved in the recognition of many different categories of objects and categoric encoding of complex visual stimuli (36) and, thus, may contribute pathologically to vivid and complex symptoms. Oishi et al. (37) compared perfusion patterns between PD with and without visual hallucinations and reported that dysfunction in the ventral pathway of the visual recognition system, especially in the right fusiform

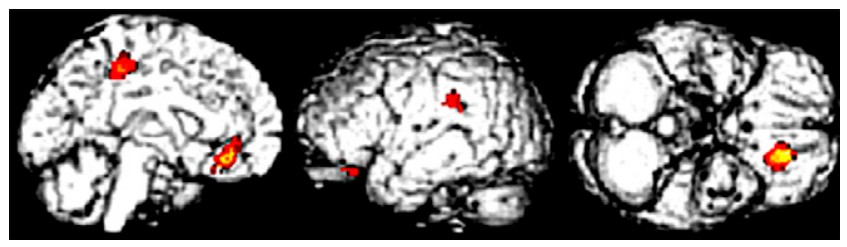

FIGURE 3. Statistical parametric maps showing correlation of increased changes in regional cerebral metabolism and increase of MMSE. 
TABLE 3

Correlation Analysis Between Increased Changes in Regional Cerebral Metabolism and Increase of MMSE

\begin{tabular}{|c|c|c|c|c|c|}
\hline \multicolumn{3}{|c|}{ Coordinate } & \multirow[b]{2}{*}{ Side } & \multirow[b]{2}{*}{ Area } & \multirow{2}{*}{$\begin{array}{c}\text { Voxel level } \\
\left(P_{\text {uncorrected }}\right)\end{array}$} \\
\hline$x$ & $y$ & $z$ & & & \\
\hline-58 & -60 & 28 & Left & Supramarginal gyrus & $<0.05$ \\
\hline-12 & 36 & -10 & Left & $\begin{array}{l}\text { Medial orbitofrontal } \\
\text { gyrus }\end{array}$ & $<0.05$ \\
\hline-10 & -39 & 48 & Left & $\begin{array}{l}\text { Middle cingulate } \\
\text { gyrus }\end{array}$ & $<0.05$ \\
\hline
\end{tabular}

gyrus, would be associated with visual processing dysfunction in PD. Mosimann et al. (38) reported the dissociation between performance in object-form and space-motion perception in patients with PDD, suggesting that visual perception associated with the ventral visual pathway was profoundly decreased. However, a decreased metabolism in the fusiform gyrus is difficult to reconcile with previous reports that $\mathrm{ChEI}$ improved visual hallucination in PDD $(3,39)$. According to a functional MRI study in patients with $\mathrm{AD}$, cholinergic modulation on visual brain responses by $\mathrm{ChEI}$ seems to be regionspecific, depending on loss of functional cholinergic cortical inputs. Bentley et al. (40) found that in patients with $\mathrm{AD}$, functionally impaired parahippocampal and precuneus regions showed a stimulus-selectivity increase of blood oxygenation level-dependant (BOLD) signals after physostigmin, whereas functionally intact fusiform cortex showed a decrease of BOLD signals, as happened in the controls. Chronic exposure to cholinesterase inhibitors may induce desensitization of the acetylcholine receptor in the fusiform gyrus, a less susceptible or functionally intact region in PDD, which leads to decreased glucose metabolism in this area after ChEI treatment.

Some limitations in our study need to be addressed. First, the sample size is small, which may influence higher improvement of MMSE scores in our study, compared with previous reports (10-12). Additionally, we could not analyze the change in cerebral metabolism between patients with cognitive improvement after ChEI treatment and those without. Second, detailed neuropsychiatric evaluations are lacking in this study, and although MMSE is a simple and universally applied scale that can be easily and rapidly performed by a clinician specializing in PDD (4), MMSE is not sensitive in detecting cognitive impairments of the frontoparietal subdomains. Therefore, we could not perform a detailed correlation analysis between the change in cognitive subdomains and cerebral metabolism.

\section{CONCLUSION}

We demonstrated that, compared with baseline, the introduction of ChEIs in patients with PDD significantly increased cerebral metabolism in the prefrontal and parietal association areas. These regions were well correlated with improvements in cognitive changes, as shown by MMSE scores, suggesting that these areas may be relevant structures for the pharmacologic response to ChEIs. A direct comparative study, with a detailed neuropsychiatric evaluation in different groups with dementia, would be necessary to uncover response patterns of cerebral metabolism in different dementia disease groups and a correlation between the change in cognitive subdomains and cerebral metabolism.

\section{ACKNOWLEDGMENTS}

This research was supported by a grant from the Stem Cell Research Center of the 21st Century Frontier Research Program (SC-4111) funded by the Ministry of Science and Technology, Republic of Korea.

\section{REFERENCES}

1. Aarsland D, Andersen K, Larsen JP, Lolk A, Nielsen H, Kragh-Sorensen P. Risk of dementia in Parkinson's disease: a community-based, prospective study. Neurology. 2001;56:730-736.

2. Aarsland D, Zaccai J, Brayne C. A systematic review of prevalence studies of dementia in Parkinson's disease. Mov Disord. 2005;20:1255-1263.

3. Caballol N, Marti MJ, Tolosa E. Cognitive dysfunction and dementia in Parkinson disease. Mov Disord. 2007;22(suppl 17):S358-S366.

4. Emre M, Aarsland D, Brown R, et al. Clinical diagnostic criteria for dementia associated with Parkinson's disease. Mov Disord. 2007;22:1689-1707.

5. Emre M. Dementia associated with Parkinson's disease. Lancet Neurol. 2003;2: 229-237.

6. Lippa CF, Duda JE, Grossman M, et al. DLB and PDD boundary issues: diagnosis, treatment, molecular pathology, and biomarkers. Neurology. 2007;68:812-819.

7. Mattila PM, Röyttä M, Lönnberg P, Marjamäki P, Helenius H, Rinne JO. Choline acetytransferase activity and striatal dopamine receptors in Parkinson's disease in relation to cognitive impairment. Acta Neuropathol (Berl). 2001;102:160-166.

8. Perry EK, Curtis M, Dick DJ, et al. Cholinergic correlates of cognitive impairment in Parkinson's disease: comparisons with Alzheimer's disease. J Neurol Neurosurg Psychiatry. 1985;48:413-421.

9. Bohnen NI, Kaufer DI, Hendrickson R, et al. Cognitive correlates of cortical cholinergic denervation in Parkinson's disease and parkinsonian dementia. J Neurol. 2006;253:242-247.

10. Emre M, Aarsland D, Albanese A, et al. Rivastigmine for dementia associated with Parkinson's disease. N Engl J Med. 2004;351:2509-2518.

11. Aarsland D, Laake K, Larsen JP, Janvin C. Donepezil for cognitive impairment in Parkinson's disease: a randomised controlled study. J Neurol Neurosurg Psychiatry. 2002;72:708-712.

12. Aarsland D, Hutchinson M, Larsen JP. Cognitive, psychiatric and motor response to galantamine in Parkinson's disease with dementia. Int J Geriatr Psychiatry. 2003;18:937-941.

13. Ceravolo R, Volterrani D, Frosini D, et al. Brain perfusion effects of cholinesterase inhibitors in Parkinson's disease with dementia. J Neural Transm. 2006;113:1787-1790.

14. Ishii K, Hosaka K, Mori T, Mori E. Comparison of FDG-PET and IMP-SPECT in patients with dementia with Lewy bodies. Ann Nucl Med. 2004;18:447-451.

15. Hughes AJ, Daniel SE, Kilford L, Lees AJ. Accuracy of clinical diagnosis of idiopathic Parkinson's disease: a clinico-pathological study of 100 cases. J Neurol Neurosurg Psychiatry. 1992;55:181-184.

16. American Psychiatric Association. Diagnostic and Statistical Manual of Mental Disorders, DSM-IV. 4th ed. Washington DC: American Psychiatric Association; 1994.

17. McKeith IG, Dickson DW, Lowe J, et al. Diagnosis and management of dementia with Lewy bodies: third report of the DLB Consortium. Neurology. 2005;65:1863-1872.

18. Lee PH, Kim JS, Shin DH, Yoon SN, Huh K. Cardiac ${ }^{123}$ I-MIBG scintigraphy in patients with drug induced parkinsonism. J Neurol Neurosurg Psychiatry. 2006; 77:372-374.

19. Tzourio-Mazoyer N, Landeau B, Papathanassiou D, et al. Automated anatomical labeling of activations in SPM using a macroscopic anatomical parcellation of the MNI MRI single-subject brain. Neuroimage. 2002;15:273-289. 
20. Tiraboschi P, Hansen LA, Alford M, et al. Cholinergic dysfunction in diseases with Lewy bodies. Neurology. 2000;54:407-411.

21. Bohnen NI, Kaufer DI, Ivanco LS, et al. Cortical cholinergic function is more severely affected in parkinsonian dementia than in Alzheimer disease: an in vivo positron emission tomographic study. Arch Neurol. 2003;60:1745-1748.

22. Teipel SJ, Drzezga A, Bartenstein P, Moller HJ, Schwaiger M, Hampel H. Effects of donepezil on cortical metabolic response to activation during ${ }^{18}$ FDGPET in Alzheimer's disease: a double-blind cross-over trial. Psychopharmacology (Berl). 2006;187:86-94.

23. Stefanova E, Wall A, Almkvist O, et al. Longitudinal PET evaluation of cerebral glucose metabolism in rivastigmine treated patients with mild Alzheimer's disease. J Neural Transm. 2006;113:205-218.

24. Potkin SG, Anand R, Fleming K, et al. Brain metabolic and clinical effects of rivastigmine in Alzheimer's disease. Int J Neuropsychopharmacol. 2001;4: 223-230.

25. Hilker R, Thomas AV, Klein JC, et al. Dementia in Parkinson disease: functional imaging of cholinergic and dopaminergic pathways. Neurology. 2005;65: 1716-1722.

26. Firbank MJ, Colloby SJ, Burn DJ, McKeith IG, O'Brien JT. Regional cerebral blood flow in Parkinson's disease with and without dementia. Neuroimage. 2003; 20:1309-1319.

27. Yong SW, Yoon JK, An YS, Lee PH. A comparison of cerebral glucose metabolism in Parkinson's disease, Parkinson's disease dementia and dementia with Lewy bodies. Eur J Neurol. 2007;14:1357-1362.

28. Emre M, Cummings JL, Lane RM. Rivastigmine in dementia associated with Parkinson's disease and Alzheimer's disease: similarities and differences. J Alzheimers Dis. 2007;11:509-519.

29. Herholz K, Bauer B, Wienhard K, et al. In-vivo measurements of regional acetylcholine esterase activity in degenerative dementia: comparison with blood flow and glucose metabolism. J Neural Transm. 2000;107:1457-1468.
30. Mori T, Ikeda M, Fukuhara R, Nestor PJ, Tanabe H. Correlation of visual hallucinations with occipital rCBF changes by donepezil in DLB. Neurology. 2006;66:935-937.

31. Rowe CC, Ng S, Ackermann U, et al. Imaging beta-amyloid burden in aging and dementia. Neurology. 2007;68:1718-1725.

32. Maetzler W, Reimold M, Liepelt I, et al. $\left[{ }^{11} \mathrm{C}\right] \mathrm{PIB}$ binding in Parkinson's disease dementia. Neuroimage. 2008;39:1027-1033.

33. Ceravolo R, Volterrani D, Tognoni G, et al. Cerebral perfusional effects of cholinesterase inhibitors in Alzheimer disease. Clin Neuropharmacol. 2004;27: 166-170.

34. Vennerica A, Shanks MF, Staff RT, et al. Cerebral blood flow and cognitive responses to rivastigmine treatment in Alzheimer's disease. Neuroreport. 2002; 13:83-87.

35. Yoshita M, Taki J, Yokoyama K, et al. Value of ${ }^{123}$ I-MIBG radioactivity in the differential diagnosis of DLB from AD. Neurology. 2006;66:1850-1854.

36. Halgren E, Dale AM, Sereno MI, Tootell RB, Marinkovic K, Rosen BR. Location of human face-selective cortex with respect to retinotopic areas. Hum Brain Mapp. 1999;7:29-37.

37. Oishi N, Udaka F, Kameyama M, Sawamoto N, Hashikawa K, Fukuyama H. Regional cerebral blood flow in Parkinson disease with nonpsychotic visual hallucinations. Neurology. 2005;65:1708-1715.

38. Mosimann UP, Mather G, Wesnes KA, O'Brien JT, Burn DJ, McKeith IG. Visual perception in Parkinson disease dementia and dementia with Lewy bodies. Neurology. 2004;63:2091-2096.

39. Burn D, Emre M, McKeith I, et al. Effects of rivastigmine in patients with and without visual hallucinations in dementia associated with Parkinson's disease. Mov Disord. 2006;21:1899-1907.

40. Bentley P, Driver J, Dolan RJ. Cholinesterase inhibition modulates visual and attentional brain responses in Alzheimer's disease and health. Brain. 2008; 131:409-424. 